\title{
ON THE DYNAMICS OF THE IRREVERSIBLE MICHAELIS-MENTEN REACTION MECHANISM
}

\author{
BERNHARD O. PALSSON \\ Department of Chemical Engineering, University of Michigan, Ann Arbor, MI 48109, U.S.A.
}

(Received 6 June 1985; accepted 23 June 1986)

\begin{abstract}
The mass action kinetic model of the irreversible Michaelis-Menten reaction mechanism is mathematically int ractable: an explicit analytical solution cannot be obtained. This difficulty is overcome by applying simplifying kinetic assumptions but a full understanding of their dynamic implications and applicability is not readily available. This paper shows how simple modal analysis can provide both a conceptually appealing insight into the reaction dynamics and justification of the commonly used quasisteady-state and quasi-equilibrium assumptions.

The key results are that the quasi-steady-state assumption is applicable when the initial enzyme concentration, $e_{0}$, is much smaller than the Michaelis constant, $K_{m}$, or when the initial substrate concentration, $s_{0}$, is much greater than $K_{\mathrm{m}}$. These results show that the commonly accepted criterion $e_{0} \ll s_{0}$ is incomplete and should be decomposed into $e_{0} \ll K_{\mathrm{m}}$ and $K_{\mathrm{m}} \ll s_{0}$. The quasi-equilibrium assumption is valid when $e_{0} \gg K_{\mathrm{m}}$ and when the rate of product formation is much slower than reversion to the substrate from the intermediate state, or $k_{2} \ll k_{-1}$. The important dimensionless parameter ratios characterizing the reaction dynamics are $e_{0} / K_{\mathrm{m}}, s_{0} / K_{\mathrm{m}}$ and $k_{2} / k_{-1}$.
\end{abstract}

\section{INTRODUCTION}

The simplest enzymatic reaction mechanism, first proposed by Henri (1903) but named after Michaelis and Menten (1913) is

$$
\underset{(s)}{\mathrm{S}}+\underset{(e)}{\mathrm{E}} \underset{k_{1}}{\rightleftharpoons} \underset{(x)}{\rightleftharpoons} \underset{k_{1}}{\mathrm{X}} \rightarrow \mathrm{E}+\underset{(p)}{\mathrm{P}}
$$

where a substrate $S$ binds reversibly to the enzyme $E$ to form the intermediate $X$, which can break down to give the product $P$ and regenerate the enzyme. The standard notation is to have the $k_{i} \mathrm{~s}$ denote appropriate rate constants and the lower case letters denote the concentrations of the corresponding chemical species. Historically the Michaelis-Menten scheme is the most important enzymatic reaction mechanism although today there is an increasing number of enzymes found that follow different kinetic mechanisms (Hill et al., 1977). A detailed account of the early history of Michaelis-Menten kinetics is found in Segal (1959).

As we will discuss below, a closed-form analytical solution to the mass action kinetic equations is attainable only by using simplifying kinetic assumptions. Two assumptions are used: the quasi-steady state assumption and the quasi-equilibrium assumption. The pioneering study by Chance (1943) on horseradish peroxidase showed, via both experimentation and computations, that the concentration profile for the intermediate complex is not stationary over a significant period of time. Chance's results motivated a series of theoretical studies into the applicability of the quasisteady-state assumption. A comprehensive review of these approximate solutions is found in Palsson (1984) and will not be repeated here. Perhaps the most complete results are found in Heineken et al. (1967) where singular perturbation theory was used to obtain separate asymptotic solutions to the fast and slow transients that this reaction normally exhibits.

In spite of these extensive efforts we still lack a comprehensive view of the parameter combinations under which the two kinetic assumptions apply. Furthermore, the mathematical techniques employed do not give good qualitative insight into reaction dynamics. This paper illustrates the use of modal analysis to help resolve these two questions. Modal analysis is suitable for this purpose since it can predict both the inherent relaxation times and how the concentrations of the chemical species move on these time scales. A particularly elegant interpretation of commonly used kinetic assumptions is obtained. Linear analysis both confirms existing results and provides new ones.

\section{MASS ACIION KINETICS}

Applying the law of mass action to the Michaelis-Menten reaction mechanism, one obtains four differential equations, one on each of the chemical species:

$$
\begin{array}{ll}
\frac{\mathrm{d} s}{\mathrm{~d} t}=-k_{1} e s+k_{-1} x, & s(t=0)=s_{0} \\
\frac{\mathrm{d} x}{\mathrm{~d} t}=k_{1} e s-\left(k_{1}+k_{2}\right) x, & x(t=0)=0 \\
\frac{\mathrm{d} e}{\mathrm{~d} t}=-k_{1} e s+\left(k_{-1}+k_{2}\right) x, & e(t=0)=e_{0} \\
\frac{\mathrm{d} p}{\mathrm{~d} t}=k_{2} x, & p(t=0)=0 .
\end{array}
$$

The initial conditions shown are for typical initial rate experiments where the substrate and free enzyme are 
mixed together at time $t=0 ; e_{0}$ and $s_{0}$ denote the initial concentration of enzyme and substrate, respectively. No mass exchange occurs with the environment and the total concentrations of enzyme and substrate stay constant. Hence we have two mass conservation equations:

$$
\begin{aligned}
& e_{0}=e+x \\
& s_{\mathrm{o}}-s+x+p .
\end{aligned}
$$

Consequently only two of eqs (2) are independent and choosing the substrate $(s)$ and the intermediate complex $(x)$ concentrations as the two independent variables the reaction dynamics are described by:

$$
\begin{aligned}
& \frac{\mathrm{d} s}{\mathrm{~d} t}=-k_{1} e_{\mathrm{o}} s+k_{-1} x+k_{1} s x, \quad s(t=0)=s_{\mathrm{O}} \\
& \frac{\mathrm{d} x}{\mathrm{~d} t}=k_{1} e_{\mathrm{o}} s-\left(k_{-1}+k_{2}\right) x-k_{1} s x, x(t=0)=0 .
\end{aligned}
$$

The major problem with this mass action kinetic model is that it is mathematically intractable (Hommes, 1962): eqs (5) and (6) can be reduced to an Abel-type differential equation whose solution cannot be obtained in a closed form. Further discussion of Abel-type differential equations and biomolecular reactions are found in Darvey et al. (1978a,b).

\section{KINETIC ASSUMPTIONS}

To get around the mathematical intractability of the mass action kinetic model, eqs (5) and (6), simplifying kinetic assumptions are applied.

The quasi-steady-state assumption (Briggs and Haldane, 1925). The rationale behind the quasisteady-state assumption is that after a rapid transient phase the intermediate $X$ reaches a quasi-stationary state in which its concentration does not change appreciably with time. Applying this assumption formally to eq. (6) gives the concentration of the intermediate complex as:

$$
x_{\mathrm{qss}}=\frac{e_{0} s}{K_{\mathrm{m}}+s}
$$

where $K_{\mathrm{m}}=\left(k_{-1}+k_{2}\right) / k_{1}$ is the well-known Michaelis constant. Substituting $x_{\text {qss }}$ into the differential equation for the substrate, eq. (5), gives the rate law

$$
\frac{\mathrm{d} s}{\mathrm{~d} t}=\frac{-k_{2} e_{0} s}{K_{\mathrm{m}}+s} \text {. }
$$

By differentiating eq. (4) one finds that $d p / d t=$ $-\mathrm{d} s / \mathrm{d} t$.

Initially the quasi-steady-state assumption was justified based on physical intuition but some justification is found within the theory of singular perturbations (Bowen et al., 1963). Equation (8) can be shown to be the first term in an asymptotic series solution derived from singular perturbation theory (Heineken et al., 1967; Meiske, 1978; see review in Palsson, 1984). Formally the time derivative of $x$ is taken to be zero which is not correct. The more appropriate statement is that the absolute value of the rate of change of $x$ is much slower than that of $s$.

The quasi-equilibrium assumption. Here one assumes that the binding step quickly reaches a quasiequilibrium state (Henri, 1903; Michaelis and Menten, 1913) where

$$
{ }_{x}^{s e}=\frac{s\left(e_{0}-x\right)}{x}=\frac{k_{-1}}{k_{1}}=K_{\mathrm{s}}, \quad \text { or } \quad x_{\mathrm{qe}}=\frac{e_{\mathrm{o}} s}{K_{s}+s}
$$

holds. Henri (1903) and Michaelis and Menten (1913) obtain the rate law

$$
\frac{\mathrm{d} p}{\mathrm{~d} t}=\frac{k_{2} e_{\mathrm{o}} s}{K_{\mathrm{s}}+s}
$$

by using relationship ( 9 ) in the differential equation for $P$. It should be noted that the same result is obtained by assuming that $\mathrm{d} s / \mathrm{d} t=0$.

Equation (10) resembles eq. (8) but they differ in two ways: first, eq. (10) describes the rate of formation of the product $\mathrm{d} p / \mathrm{d} t$ as opposed to the disappcarance of the substrate $\mathrm{d} s / \mathrm{d} t$, and second, it contains the dissociation constant $K_{\mathrm{s}}$ as opposed to the Michaelis constant $K_{\mathrm{m}}$. A differential equation for the substrate is obtained differentiating eqs (4) and (9) as

$$
\frac{\mathrm{d} s}{\mathrm{~d} t}=\frac{-k_{2} e_{0} s}{K_{\mathrm{s}}+s+\frac{e_{\mathrm{o}} K_{\mathrm{s}}}{K_{\mathrm{s}}+s}}
$$

and therefore $\mathrm{d} p / \mathrm{d} t \neq-\mathrm{d} s / \mathrm{d} t$.

\section{LINEAR ANALYSIS}

Linearizing the mass action kinetic model yields an approximate description of reaction dynamics in the vicinity of the point around which the linearization is carried. The linear description is of course only an approximation to the exact solution but as we will see. is valuable for providing qualitative information about reaction dynamics and a guide to model simplification.

Formally the linearization is carried out by expanding the non-linear right-hand sides of eqs (5) and (6) into a Taylor series and truncating the series after the linear term:

$$
f(\mathbf{y})=f\left(\mathbf{y}_{\mathrm{ref}}\right)+J\left(\mathbf{y}-\mathbf{y}_{\mathrm{ref}}\right)+\cdots
$$

where $f$ represents the non-linear right-hand sides of eqs (5) and (6), $y$ is the concentration vector $(s, x)^{\mathrm{t}}$ and $J$ is the Jacobian matrix $\left(j_{i k}=\mathrm{d} f_{i} / \mathrm{d} y_{k}\right)$. Linearizing the Michaelis-Menten kinetic equations gives a $2 \times 2$ matrix description

$$
\frac{\mathrm{d} \mathbf{y}^{\prime}}{\mathrm{d} t}=f\left(\mathbf{y}_{\mathrm{ref}}\right)+J \mathbf{y}^{\prime}, \quad \mathbf{y}^{\prime}=\left(s^{\prime}, x^{\prime}\right)^{t}
$$

where the prime denotes that the variables are in terms of deviation from the reference conditions (i.e. $s^{\prime}=s$ $-s_{\text {ref }}$ and $x^{\prime}=x-x_{\text {ref }}$ ). The Taylor series can be obtained around any reference point. The Jacobian 
matrix is

$$
J=\left(\begin{array}{cc}
-k_{1} e_{0} & k_{-1}+k_{1} s_{0} \\
k_{1} e_{0} & -\left(k_{2}+k_{-1}+k_{1} s_{0}\right)
\end{array}\right)
$$

The reference state here is taken to be the initial condition $s_{\text {ref }}=s_{0}, x_{\text {ref }}=0$, since we are interested in how the reaction moves away from the initial conditions.

The properties of $J$ describe the dynamic characteristics of the linearized model. The properties of $J$ that are of interest are basically two:

(a) The eigenvalues, $\lambda_{1}\left(=-1 / \tau_{1}\right)$ and $\lambda_{2}\left(=-1 / \tau_{2}\right)$ give the two relaxation times $\left(\tau_{1}, \tau_{2}\right)$, intrinsic to the reaction. The eigenvalues are the two roots of the characteristic equation

$$
\lambda^{2}-\operatorname{tr}(J) \lambda+\operatorname{det}(J)=0
$$

where $\operatorname{tr}(J)=-k_{1}\left(K_{m}+e_{0}+s_{0}\right)$ is the trace of the Jacobian matrix and $\operatorname{det}(J)=k_{1} k_{2} e_{0}$ is the determinant.

The two relaxation times are normally separated by more than one order of magnitude (Palsson, 1984; Palsson and Lightfoot, 1984) leading to a dynamic response that contains two distinct time scales: a slow and a fast phase, which are normally referred to as the stationary and the transient phase, respectively

(b) The normal modes, $m=\left(m_{1}, m_{2}\right)^{t}$. The modes are abstract mathematical quantities that are, by definition, dynamically independent. The modes will give us information about how the concentrations of $S$ and $X$ move on the intrinsic time scales.

The key point here is that the modes are dynamically independent: motion of mode 1 does not disturb the motion of mode 2 . Then if the time constants are well separated and the smaller time constant represents dynamics that are faster than the time scales of interest, then one can relax the dynamic motion of the faster mode without changing the system behaviour significantly on the slower time scale. This key feature forms the basis for the interpretation of reaction dynamics and system simplification that follows.

The modes are related to the concentration variables through the modal matrix $M^{-1}$, which is comprised of the eigenrows, as

$$
\left(\begin{array}{l}
m_{1} \\
m_{2}
\end{array}\right)=M^{-1}\left(\begin{array}{l}
s \\
x
\end{array}\right)=\left(\begin{array}{ll}
1 & c_{1} \\
c_{2} & 1
\end{array}\right)\left(\begin{array}{l}
s \\
x
\end{array}\right)
$$

where $c_{1}$ and $c_{2}$ are the independent elements of the eigenrows, $\mathbf{u}_{1}=\left(1, c_{1}\right)$ and $\mathbf{u}_{2}=\left(c_{2}, 1\right)$. The eigenrows are defined as:

$$
\mathbf{u}_{i}\left(J-\lambda_{i} I\right)=0 .
$$

The relationship between the motion of the modes and the concentration variables is given by differentiation of eq. (16):

$\frac{\mathrm{d} m_{1}}{\mathrm{~d} t}=\frac{\mathrm{d} s}{\mathrm{~d} t}+c_{1} \frac{\mathrm{d} x}{\mathrm{~d} t} \quad($ moves on time scale 1$)$ $\frac{\mathrm{d} m_{2}}{\mathrm{~d} t}=c_{2} \frac{\mathrm{d} s}{\mathrm{~d} t}+\frac{\mathrm{d} x}{\mathrm{~d} t} \quad$ (moves on time scale 2).

Since the modes are dynamically independent their concentration composition give us an important insight into the reaction dynamics by allowing us to examine the motion of the concentration variables on both time scales. The constants $c_{1}$ and $c_{2}$ are measures of the dynamic interactions between the two dynamic variables, $s$ and $x$, since they indicate their relative contribution to the motion on the two time scales.

\section{ORDER OF MAGNITUDE ANALYSIS}

It can be shown (Palsson, 1984) that when the ratio $\operatorname{det}(J) / \operatorname{tr}(J)^{2}$ is small, the time constants and the interaction coefficients, $c_{1}, c_{2}$, take the limiting values

$$
\begin{array}{ll}
\tau_{1} \rightarrow \frac{-\operatorname{tr}(J)}{\operatorname{det}(J)} \quad c_{1} \rightarrow \frac{\frac{j_{11}^{2}}{j_{21}}-j_{12}}{j_{11}+j_{22}} \\
\tau_{2} \rightarrow \frac{-1}{\operatorname{tr}(J)} & c_{2} \rightarrow \frac{j_{21}}{j_{22}} .
\end{array}
$$

These results are obtained by using the first column of $J$. Identical results are derived by using the second column since the matrix $(J-\lambda I)$ is singular. The ratio $\operatorname{det}(J) / \operatorname{tr}(J)^{2}$ is:

$$
\frac{\operatorname{det}(J)}{\operatorname{tr}(J)^{2}}=\frac{\frac{k_{2} / k_{-1}}{1+k_{2} / k_{-1}} \frac{e_{0}}{K_{m}}}{\left(1+\frac{e_{0}}{K_{m}}+\frac{s_{0}}{K_{m}}\right)^{2}}
$$

This ratio is small under four parameter combinations:
(a) when $e_{0} \ll K_{m}$,
(b) when $s_{0} \gg K_{m}$,
(c) when $e_{\mathrm{o}} \gg K_{\mathrm{m}}$, and
(d) when $k_{2} \ll k_{-1}$,

or combinations thereof. The limiting values of the $\tau_{i} s$ and $c_{i}$ s are shown in Table 1.

Two limits are of particular importance: limit (a), since the total enzyme concentration is much less than the Michaelis constant for typical batch kinetic experiments (Srere, 1967, 1970; Masters, 1977), and limit (d), since this situation is found for many enzymes (Hammes and Schimmel, 1970; Cleland, 1975; Albery and Knowles, 1976, 1977).

\section{Dynamic behaviour}

Limits (a)-(d) represent the fundamental functional dependences of the time-scale separation on the intrinsic dimensionless property ratios (see Section 6). Combinations of the above limits will also produce time-scale separation. One particular combination, $e_{0} \ll s_{0}$, is of historic importance and this situation will be discussed in Section 7.

We will now use modal analysis to interpret the reaction dynamics. The predictions obtained are confirmed by direct numerical integration in Section 7 below. 
Table 1. Limiting values of the time constants and the interaction coefficients, $a=k_{2} / k_{-1}, b=e_{0} / K_{m}$ and $c=s_{\mathrm{o}} / K_{\mathrm{m}}$

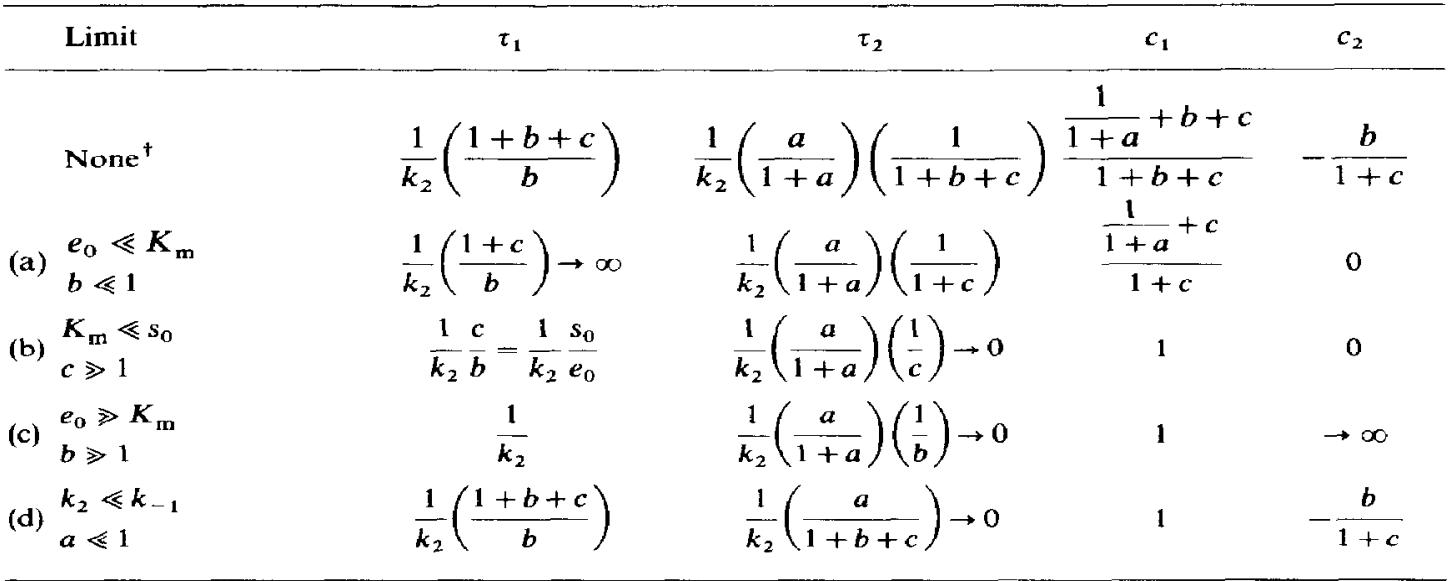

† General expressions derived by using the first column of the Jacobian matrix. Same results for the limiting expressions are found by using the second column of $\boldsymbol{J}$.

Limit $(a), e_{0} \ll K_{\mathrm{m}}$. Here the limiting value of $c_{2}$ is zero and the modes can be written as:

$$
\begin{array}{ll}
\frac{\mathrm{d} m_{1}}{\mathrm{~d} t}=\frac{\mathrm{d}\left(s+c_{1} x\right)}{\mathrm{d} t} & \text { (slow mode) } \\
\frac{\mathrm{d} m_{2}}{\mathrm{~d} t}=\frac{\mathrm{d} x}{\mathrm{~d} t} & \text { (fast mode). }
\end{array}
$$

Consequently we obtain the following interpretation of reaction dynamics:

The fast motion. Only $x$ moves significantly on the faster time scale. The concentration of $X$ will relax towards a quasi-steady-state value $x_{q s s}$ given by the solution of $\mathrm{d} x / \mathrm{d} t=0$, eq. (7).

The slow motion. Once the rapid motion has relaxed the two concentrations move in a fixed relationship to one another given by eq. (7), which leads to the quasi-steady-state approximation.

Hence modal analysis yields an elegant interpretation of the reaction dynamics: the fast transients described by mode 2 are only those of $x$ and if one wishes to ignore these dynamics without disturbing the slow motion one can apply $\mathrm{d} m_{2} / \mathrm{d} t=\mathrm{d} x / \mathrm{d} t=0$. Hence the quasi-steady-state assumption should apply.

Limit $(b), K_{\mathrm{m}} \ll s_{\mathrm{U}}$. This leads to a similar simplification as occurs in limit (a) and zeroth-order kinetics. When $K_{\mathrm{m}} \ll s_{0}$, the composition of the modes is

$$
\begin{array}{ll}
\frac{\mathrm{d} m_{1}}{\mathrm{~d} t}=\frac{\mathrm{d}(s+x)}{\mathrm{d} t}=-\frac{\mathrm{d} p}{\mathrm{~d} t} & \text { (slow mode) } \\
\frac{\mathrm{d} m_{2}}{\mathrm{~d} t}=\frac{d x}{\mathrm{~d} t} . & \text { (fast mode) }
\end{array}
$$

The fast motion. As for limit (a), the fast motion is essentially that of $x$.
The slow motion. The motion on the slower time scale is that of a "pool" of $s+x$.

The differential equation describing the slow motion is

$$
\frac{\mathrm{d} m_{1}}{\mathrm{~d} t}=\frac{\mathrm{d}(\mathrm{s}+x)}{\mathrm{d} t}=-\frac{\mathrm{d} p}{\mathrm{~d} t}=-k_{2} x=\frac{-k_{2} e_{0} s}{K_{\mathrm{m}}+\mathrm{s}} .
$$

The value of $x$ used here is the one given by eq. (7)

Limit (c), $e_{0} \gg K_{\mathrm{m}}$. The modes become

$$
\begin{array}{ll}
\frac{\mathrm{d} m_{1}}{\mathrm{~d} t}=\frac{\mathrm{d}(s+x)}{\mathrm{d} t} & \\
\frac{\mathrm{d} m_{2}}{\mathrm{~d} t}=\frac{\mathrm{d} s}{\mathrm{~d} t} & \text { (flow motion) }
\end{array}
$$

in this limit. The interpretation of the reaction dynamics is as follows:

The fast motion. The opposite situation to limits (a) and (b) occurs: the fast motion is essentially that of the substrate. Relaxing differential eq. (5) leads to the equilibrium relationship for the binding step, eq. (9).

The slow motion. As for limit (b) the slow motion is that of the pool $s+x$.

Based on linear analysis we expect a rapid motion towards the equilibrium state by motion of $s$. The quasi-equilibrium assumption should give good results.

If the quasi-equilibrium assumption is invoked one can no longer use $s_{0}$ as the initial value for $s$ since it moves significantly on the faster time scale. A new initial value must be provided. By assuming that $\boldsymbol{m}_{\mathrm{t}}$ stays at its initial value during the relaxation of $m_{2}$ one obtains

$$
m_{1}=s_{\mathrm{qe}}+x_{\mathrm{qe}}=s_{\mathrm{o}} .
$$

By combining this result with the equilibrium relation- 
ship for the binding step one gets

$$
s_{\mathrm{qe}}^{2}+\left(K_{\mathrm{s}}+e_{\mathrm{o}}-s_{0}\right) s_{\mathrm{qe}}-s_{0} K_{\mathrm{s}}=0
$$

The appropriate initial vlaue is the positive root of eq. (27).

Limit $(d), k_{2} \ll k_{-1}$. In this limit the composition of the modes is

$$
\begin{array}{ll}
\frac{\mathrm{d} m_{1}}{\mathrm{~d} t}=\frac{\mathrm{d}(s+x)}{\mathrm{d} t} \quad \text { (slow mode) } \\
\frac{\mathrm{d} m_{2}}{\mathrm{~d} t}=\frac{\mathrm{d}\left(c_{2} s+x\right)}{\mathrm{d} t} \quad \text { (fast mode). }
\end{array}
$$

Relaxing the dynamics of $m_{2}$ leads to the equilibrium relationship for the substrate binding step and the following interpretation of the reaction dynamics is obtained:

The fast motion. The fast mode describes motion towards the equilibrium state for the binding step, by motion of both $s$ and $x$.

The slow motion. Again the slow motion is that of the pool $s+x$.

Hence, we expect to see a two-phase transient response: a rapid approach to a quasi-equilibrium state for the binding step and a slow motion in which $s$ and $x$ are dynamically equivalent. Again, modal analysis predicts the use of the quasi-equilibrium assumption and gives us a clear physical interpretation of the reaction dynamics.

As for limit (c), one cannot use $s(t=0)=s_{0}$ and $x(t=0)=0$ as initial conditions since both variables move significantly on the faster time scale. The initial conditions have to be adjusted using eq. (27).

\section{SCALING}

The above analysis suggests that there are three dimensionless property ratios of interest:

$$
a=k_{2} / k_{-1}, \quad b=e_{0} / K_{\mathrm{m}}, \quad c=s_{0} / K_{\mathrm{m}} .
$$

The first dimensionless group, $a$, is a ratio consisting only of kinetic constants, $k_{2} / k_{-1}$. This ratio has been called the "stickiness number" (Palsson, 1984), since a substrate is said to stick well to an enzyme if $k_{2}>k_{-1}$; once $\mathrm{X}$ is formed it is more likely to break down to yield the product than to revert back to the substrate.

The second dimensionless number $e_{0} / K_{m}$, is a dimensionless concentration parameter; the total enzyme concentration relative to the Michaelis constant. This quantity varies from one situation to another and takes particularly different values under in vitro and in vivo conditions. In vitro the enzyme concentrations used are several orders of magnitude lower than the $K_{\mathrm{m}}$ values (Srere, 1967, 1970; Masters, 1977). Typically, in vivo enzyme concentrations can approach the same order of magnitude as $K_{\mathrm{m}}$.
The third dimensionless ratio, $s_{0} / K_{m}$, is the initial condition for the substrate concentration. Typical values for this ratio for both in vivo and in vitro situations are of the order of unity.

A natural choice of reference scales for the concentrations are:

$$
\text { for the substrate, } \quad \sigma=s / K_{\mathrm{m}}
$$

and for the intermediate, $\chi=x / e_{0}$.

Casting the differential equations into dimensionless form gives

$$
\begin{array}{rlrl}
\frac{\mathrm{d} \sigma}{\mathrm{d} \tau} & =-\sigma+\frac{1}{1+a} \chi+\chi \sigma, & \sigma_{0} & =c \\
b \frac{\mathrm{d} \chi}{\mathrm{d} \tau} & =\sigma-\chi-\chi \sigma, & \chi_{0}=0
\end{array}
$$

where time is scaled as $\tau=k_{1} e_{0} t$. The quasi-steadystate and quasi-equilibrium concentrations of the intermediate in scaled variables are:

$$
\begin{aligned}
& \chi_{\mathrm{qss}}=\frac{\sigma}{1+\sigma} \\
& \chi_{\mathrm{qe}}=\frac{\sigma}{\frac{1}{1+a}+\sigma}
\end{aligned}
$$

\section{NUMERICAL SOLUTIONS}

The full dynamic description of the kinetics of the reaction, eqs (5) and (6), can be obtained by direct numerical integration. The numerical solutions are obtained using the EPISODE ordinary differential equation integrator (Byrne and Hindmarsh, 1975). Here we use the equations in their more compact dimensionless form, eq. (31).

The results are most conveniently shown on a phase portrait along with the transient response of the concentrations on both the fast and slow time scales. The following results ascertain the predictions from full linear analysis.

Limit $(a)$, $e_{\mathrm{o}} \ll K_{\mathrm{m}}$.

Figure 1 shows the full numerical solution for $b$ $=0.01$ and the other groups assume the value of unity.

The phase plane. The phase plane is shown in Fig. 1 (a) and it shows how the reaction rapidly approaches the quasi-steady-state line and then moves along that line towards the equilibrium point, $\sigma=0, \chi=0$.

The fast motion. Figure 1(b) shows the changes in the concentrations during the faster time scale. As predicted by modal analysis, the intermediate concentration exhibits a significant fast motion while the substrate does not move far from its initial value.

The slow motion. The changes in the concentrations during the slower time scale are shown in Fig. 1 (c). 


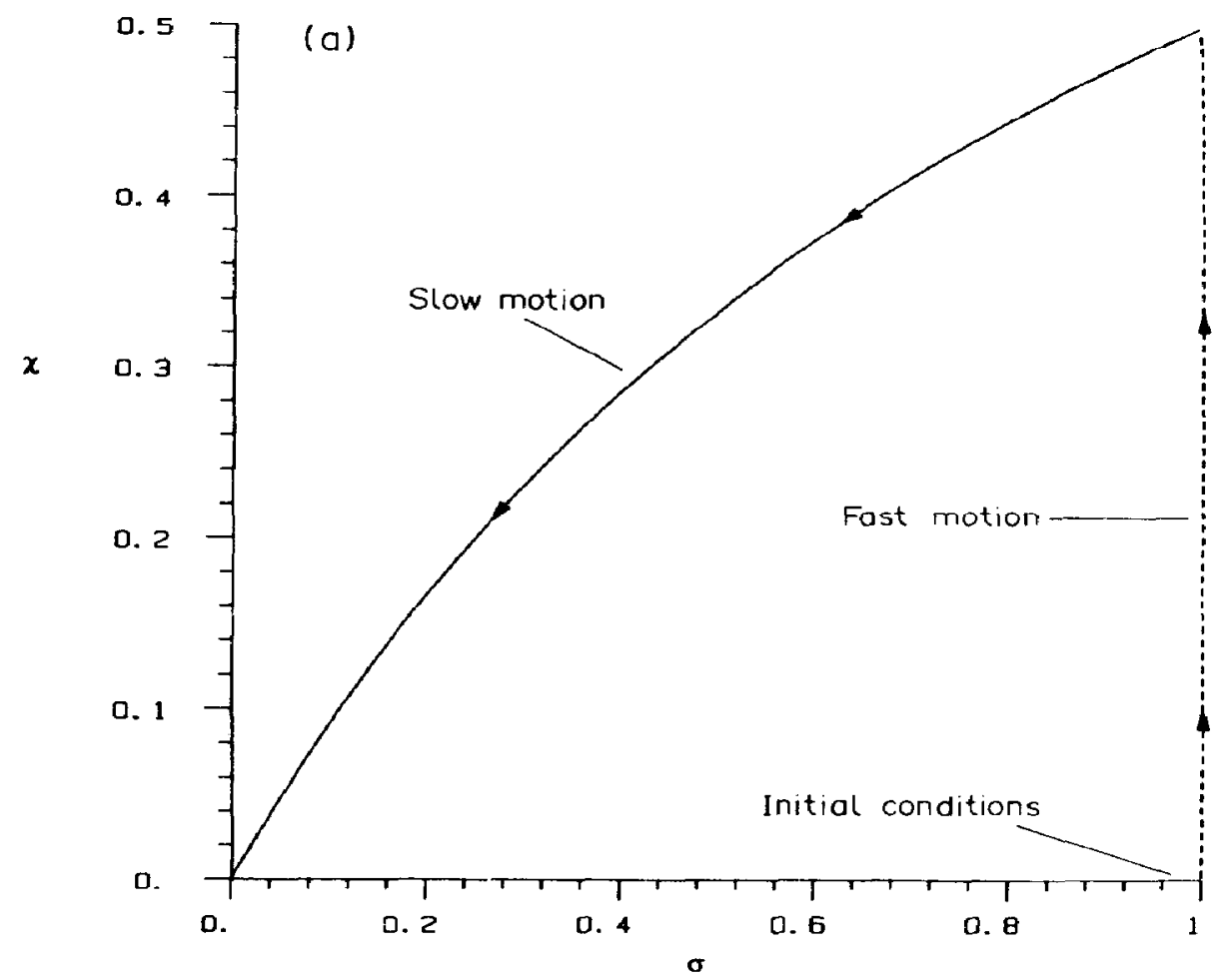

(b)

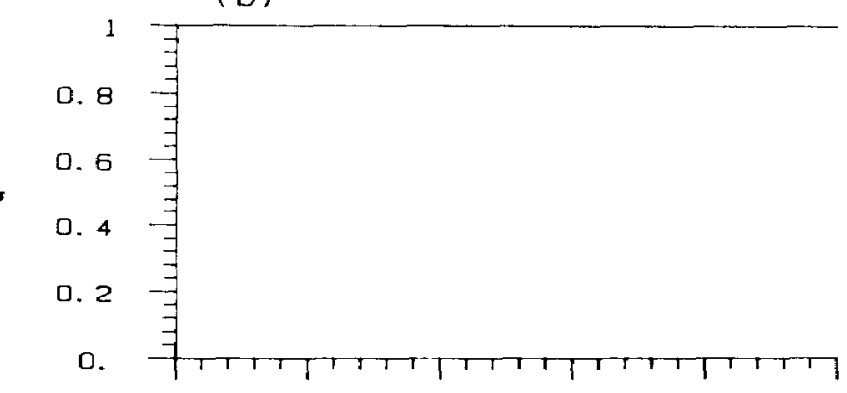

(c)
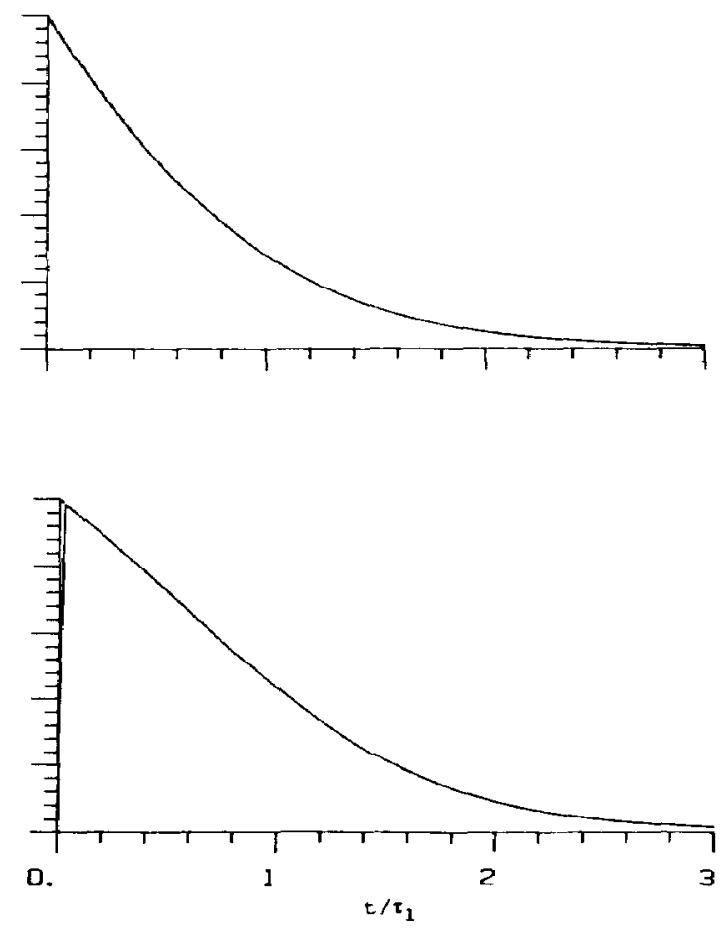

Fig. 1. Dynamic behaviour of the Michaelis-Menten reaction mechanism for $k_{2} / k_{-1}=1, e_{n} / K_{\mathrm{m}}=0.01$, $s_{0} / K_{\mathrm{m}}=1$. (a) The phase plane (solid line, quasi-steady-state solution; dashed line, full numerical solution). (b) The fast transients. (c) The slow transients (solid line, full numerical solution; dashed line, quasi-stcadystate solution). 
Both $\sigma$ and $\chi$ decay towards zero. During the decay process, $\chi$ is in a quasi-stationary state and the motion of $\sigma$ drives the reaction dynamics. The quasi-steadystate solution gives a good description of the events on the slower time scale.

Limit $(b), s_{\mathrm{O}} \gg K_{\mathrm{m}}$. Figure 2 shows the reaction dynamics when $c=50$ and the other two groups are unity.

The phase plane. Figure 2(a) shows the motion in the phase plane. As for limit (a), the reaction approaches the quasi-steady-state line primarily by change in the intermediate concentration. After the fast transients relax, the reaction is in the zeroth-order regime.

The fast motion. Figure 2(b) shows that, as for limit (a), the fast motion essentially is the motion of $x$ towards a quasi-steady state.

The slow motion. The slow transients, Fig. 2(c), are those of a zeroth-order reaction where the intermediate is in a quasi-steady state.

\section{Limit $(c), e_{0} \gg K_{\mathrm{m}}$}

The reaction dynamics when $b=100$ and $a=c=1$ are shown in Fig. 3.

The phase plane. The phase portrait is shown in Fig. 3 (a). As predicted in Section 5, a motion towards the quasi-equilibrium line primarily by change in the substrate concentration is found. Then the reaction path lies along the quasi-equilibrium line.

The fast motion. The fast dynamics are essentially those of the substrate as shown in Fig. 3(b).

The slow motion. After the fast dynamics have relaxed, $\sigma$ and $\chi$ move in a quasi-cquilibrium state, Fig. 3 (c). The transients on the slower time scale are adequately described using the quasi-equilibrium assumption if the initial conditions are adjusted according to eq. (27).

Limit $(d), k_{2} \ll k_{-1}$

The reaction dynamics for $a=0.01$ and $b=c=1$ are shown in Fig. 4.

The phase plane. The phase plane, Fig. 4(a) shows how the reaction moves towards the quasi-equilibrium line by motion of both $\sigma$ and $\chi$. Then once in the quasiequilibrium state the reaction moves along the quasiequilibrium line towards the equilibrium point. The quasi-steady-state and quasi-equilibrium line are essentially identical in this limit, cf. eq. (32).

The fast motion. Figure 4(b) shows the transient changes in the concentrations during the approach to the quasi-equilibrium state. Both concentrations undergo significant changes.
The slow motion. Figure 4(c) shows the slow transients of the reaction and the quasi-equilibrium solution is adequate to describe these transients if the initial conditions are properly adjusted, eq. (27).

Comment on the criterion $e_{0} \ll s_{\mathrm{o}}$

The commonly accepted and quoted criterion for the applicability of the quasi-steady-state assumption is that the initial concentration of the enzyme must be much smaller than that of the substrate. Our analysis above indicates that this is an incomplete criterion and the proper dimensionless groups to use are $b$ and $c$. Limit (a) is still valid even though the value of $s_{0}$ is much below $K_{\mathrm{m}}$. Figure 5 shows the reaction dynamics for $a=1, b=c=0.01$ which is analogous to Fig. 1 except that the initial substrate concentration is now 100 times smaller than $K_{\mathrm{m}}$. In other words, we have that $e_{0}=s_{0} \ll K_{m}$ and as demonstrated in Fig. 5, the quasi-equilibrium assumption is applicable.

\section{Conclusions}

Through simple modal analysis we are able to conceptualize and justify the applicability of the kinetic assumptions used to simplify the mass action kinetic model of the Michaclis-Menten reaction mechanism. Linear analysis predicts the following basic parameter combination for the validity of the kinetic assumptions:

for the quasi-steady state assumption, $e_{0} \ll K_{\mathrm{m}}$, $K_{\mathrm{m}} \ll s_{0}$;

for the quasi-equilibrium assumption, $e_{0} \geqslant K_{\mathrm{m}}$, $k_{2} \ll k_{-1}$.

\section{Discussion}

Full linear analysis, including the consideration of the normal modes, gives the capability to resolve some long-standing questions about the kinetic behaviour of the Michaelis-Menten reaction mechanism. The parameter combinations under which the commonly used quasi-steady-state and quasi-equilibrium assumptions apply are predicted by modal analysis and confirmed via full numerical integration. Furthermore, linear analysis leads to an elegant interpretation and a clear conceptualization of the reaction dynamics.

Our analysis shows that the commonly accepted criterion $e_{0} \ll s_{0}$ for the applicability of the quasisteady-state assumption is incomplete. The assumption of quasi-stationary behaviour is found to depend on the relative magnitude of $e_{0}$ and $s_{0}$ to $K_{\mathrm{m}}$ [limits (a) and (b)], both of which are concentration-dependent effects. For instance, we can have the condition $e_{0}=s_{0} \ll K_{\mathrm{m}}$ and still apply the quasi-steady-state assumption (cf. Fig. 5). What is of particular importance here is that as long as the initial enzyme concentration is small compared to the Michaelis constant, the quasi-steady-state solution is valid regardless of the initial concentration of the substrate. As pointed out in Section $5, e_{0} \ll K_{m}$ is a commonly found situation for initial rate experiments. However, in vivo the concen- 


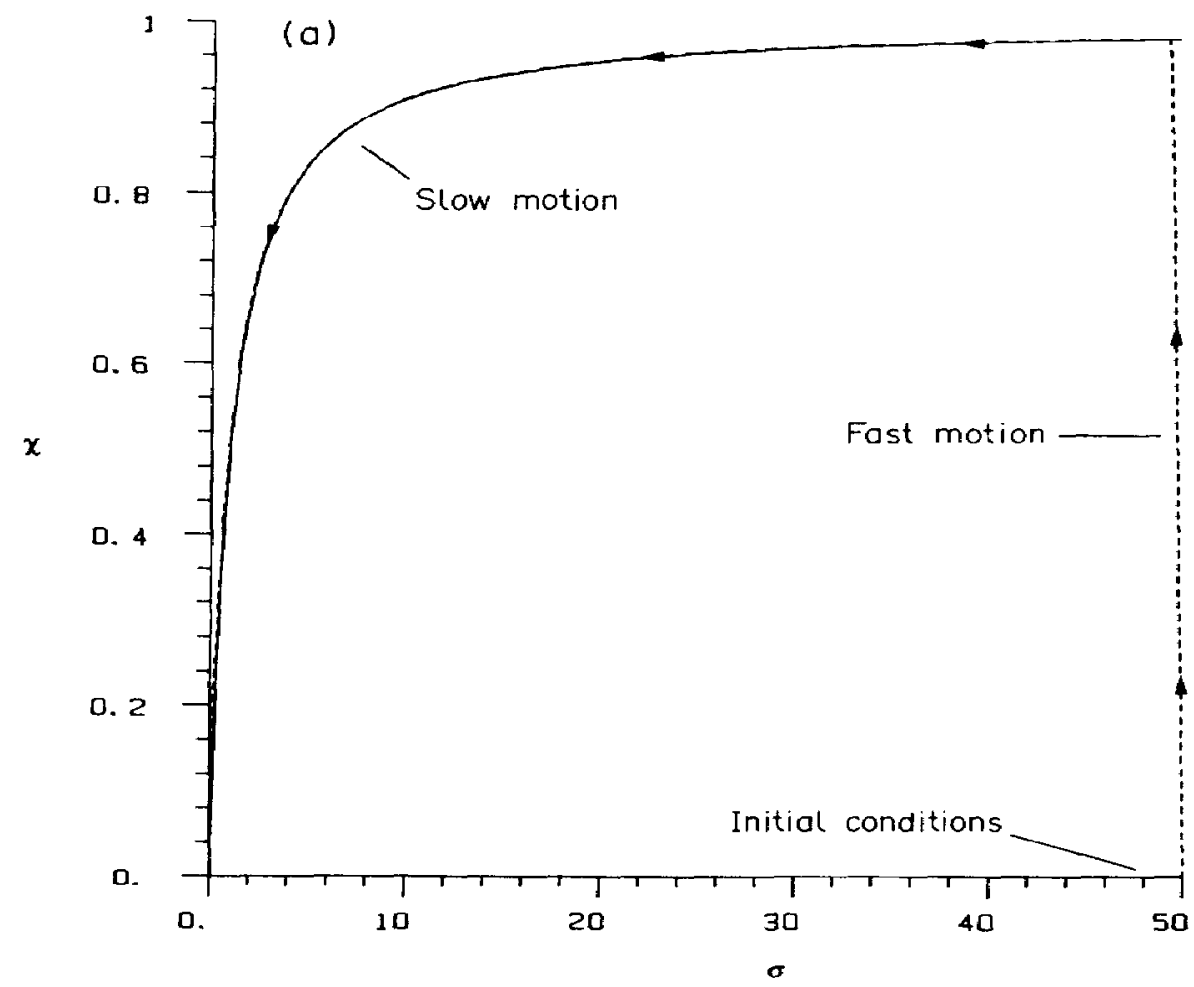

$\sigma$

(b)

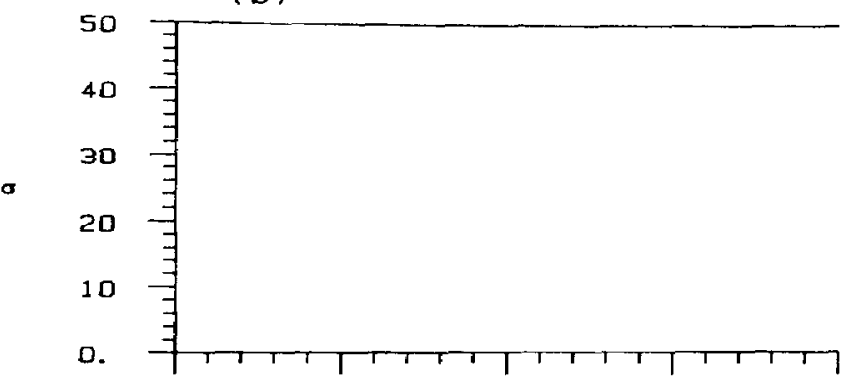

(c)

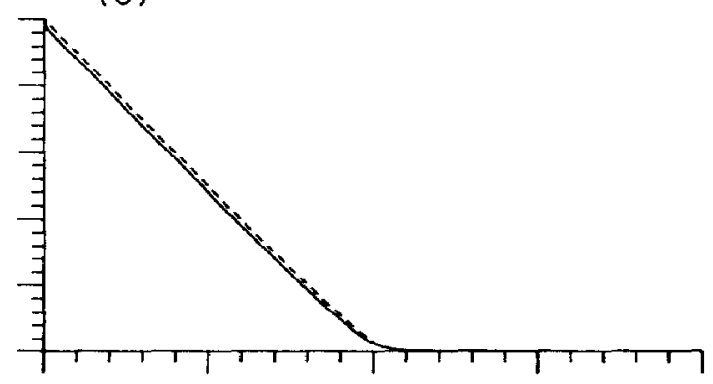

$x$
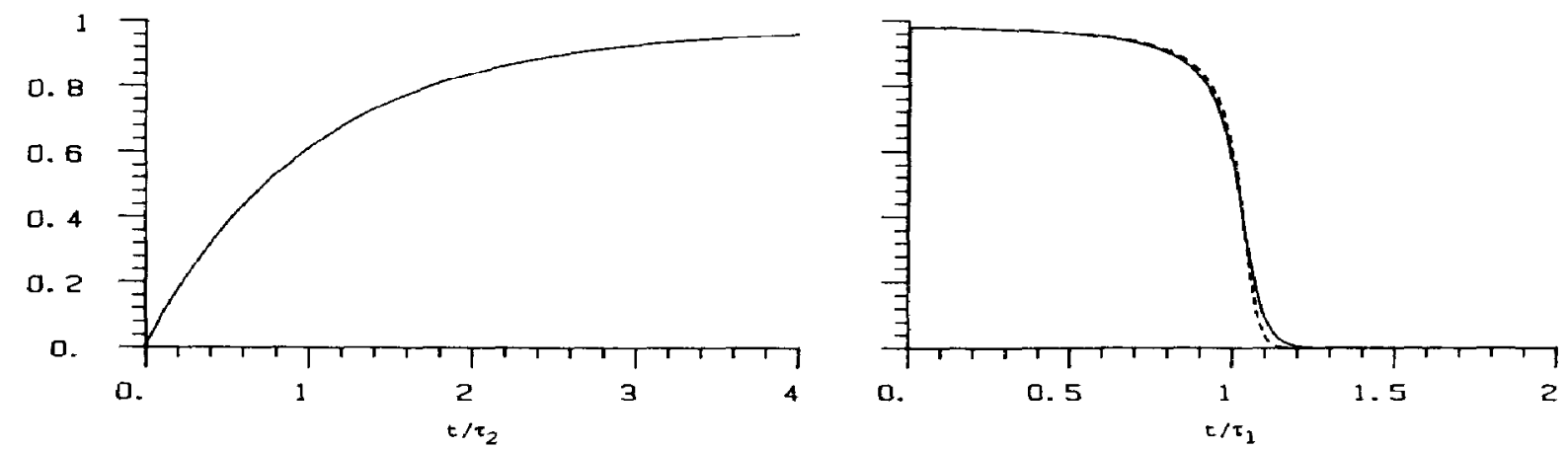

Fig. 2. Dynamic behaviour of the Michaelis-Menten reaction mechanism for $k_{2} / k_{-1}=1, e_{0} / K_{\mathrm{m}}=0.01$, $s_{0} / K_{\mathrm{m}}=50$. (a) The phase plane (solid line, quasi-steady-state solution; dashed line, full numerical solution). (b) The fast transients. (c) The slow transients (solid line, full numerical solution; dashed line, quasi-steadystate solution). 


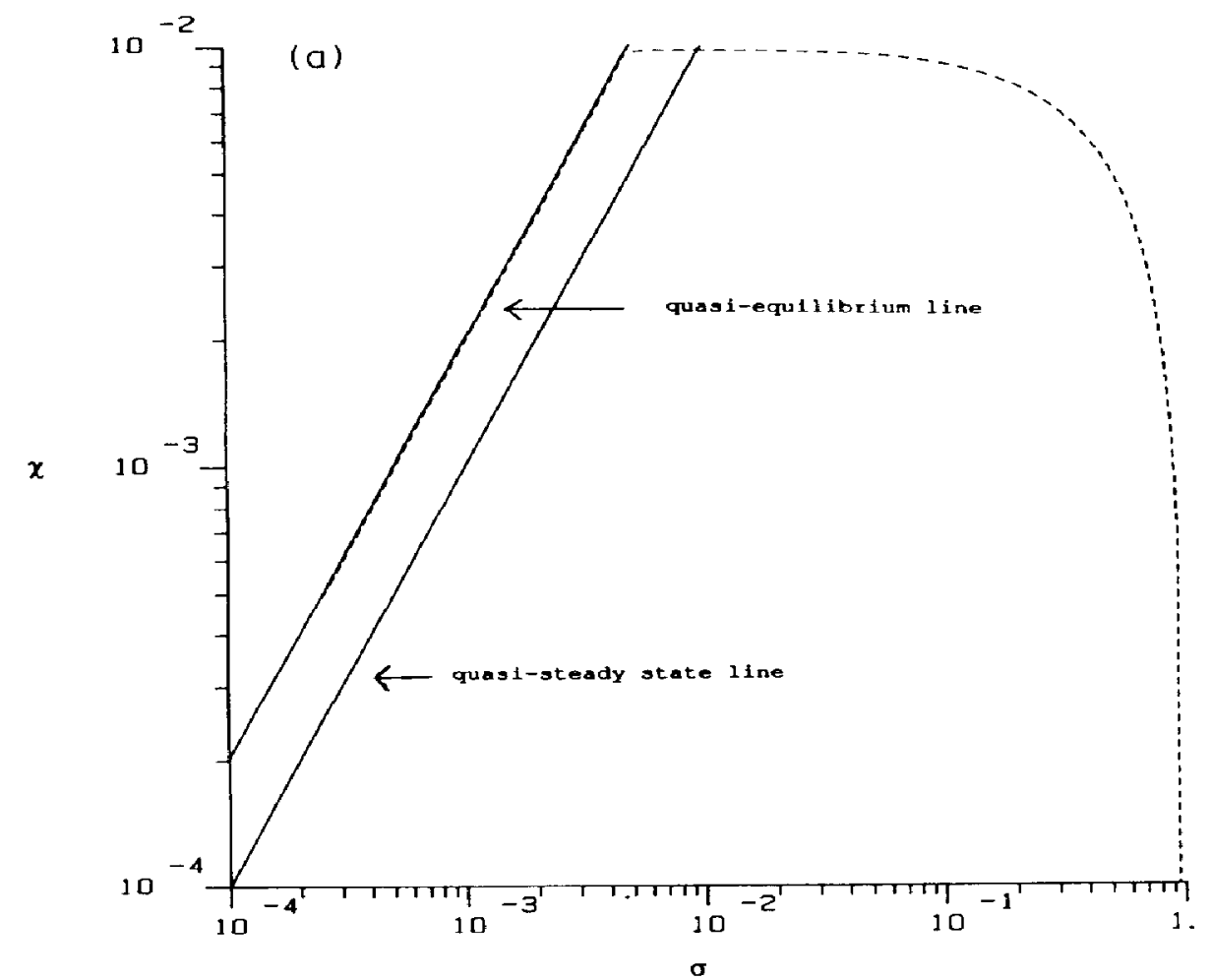

o
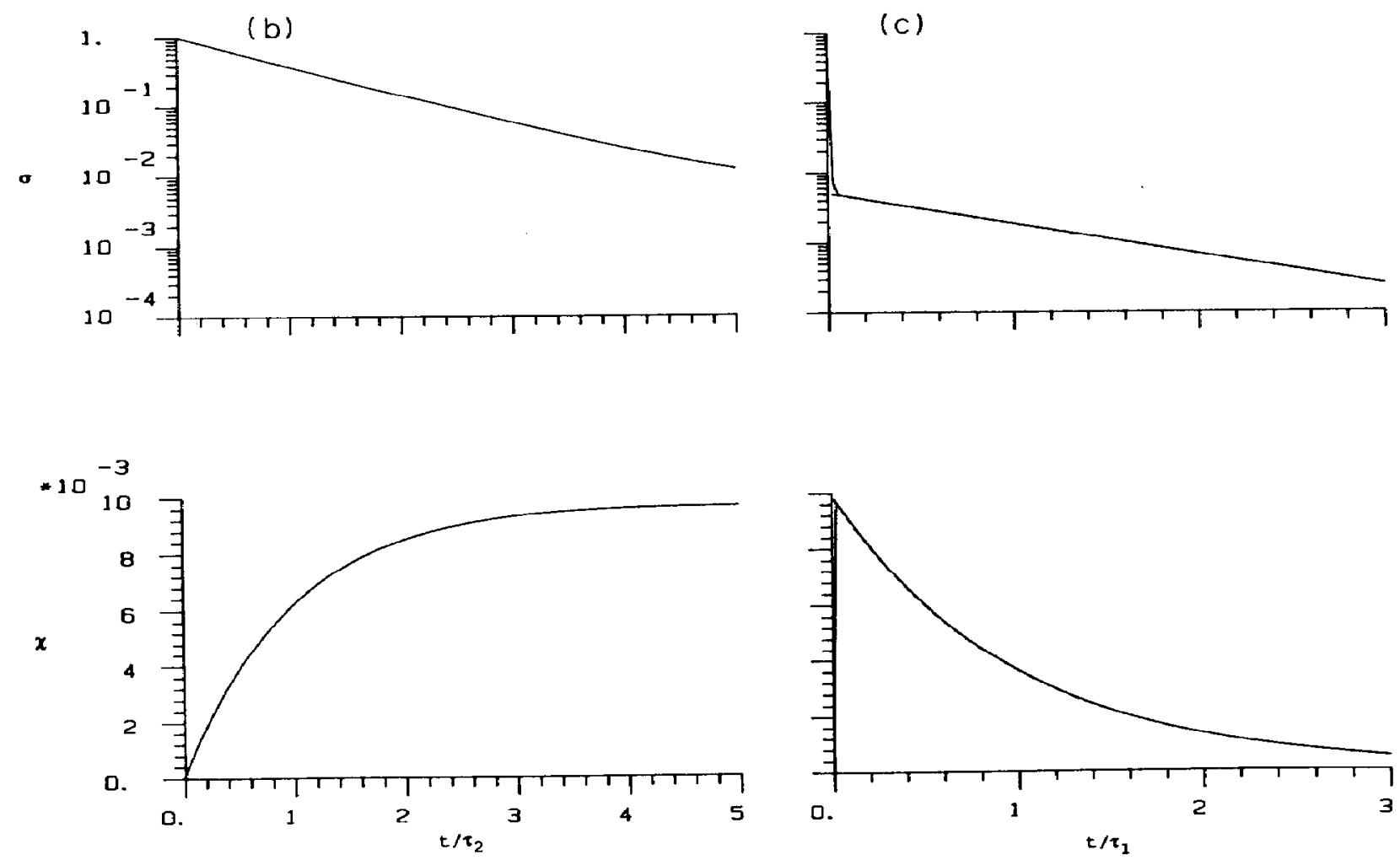

Fig. 3. Dynamic behaviour of the Michaelis-Menten reaction mechanism for $k_{2} / k_{-1}=1, \epsilon_{0} / K_{\mathrm{m}}=100$, $s_{\mathrm{o}} / K_{\mathrm{m}}=1$. (a) The phase plane (solid lines, quasi-equilibrium and quasi-steady-state solutions; dashed line, full numerical solution). (b) The fast transients. (c) The slow transients (solid line, full numerical solution; dashed line, quasi-equilibrium solution). 


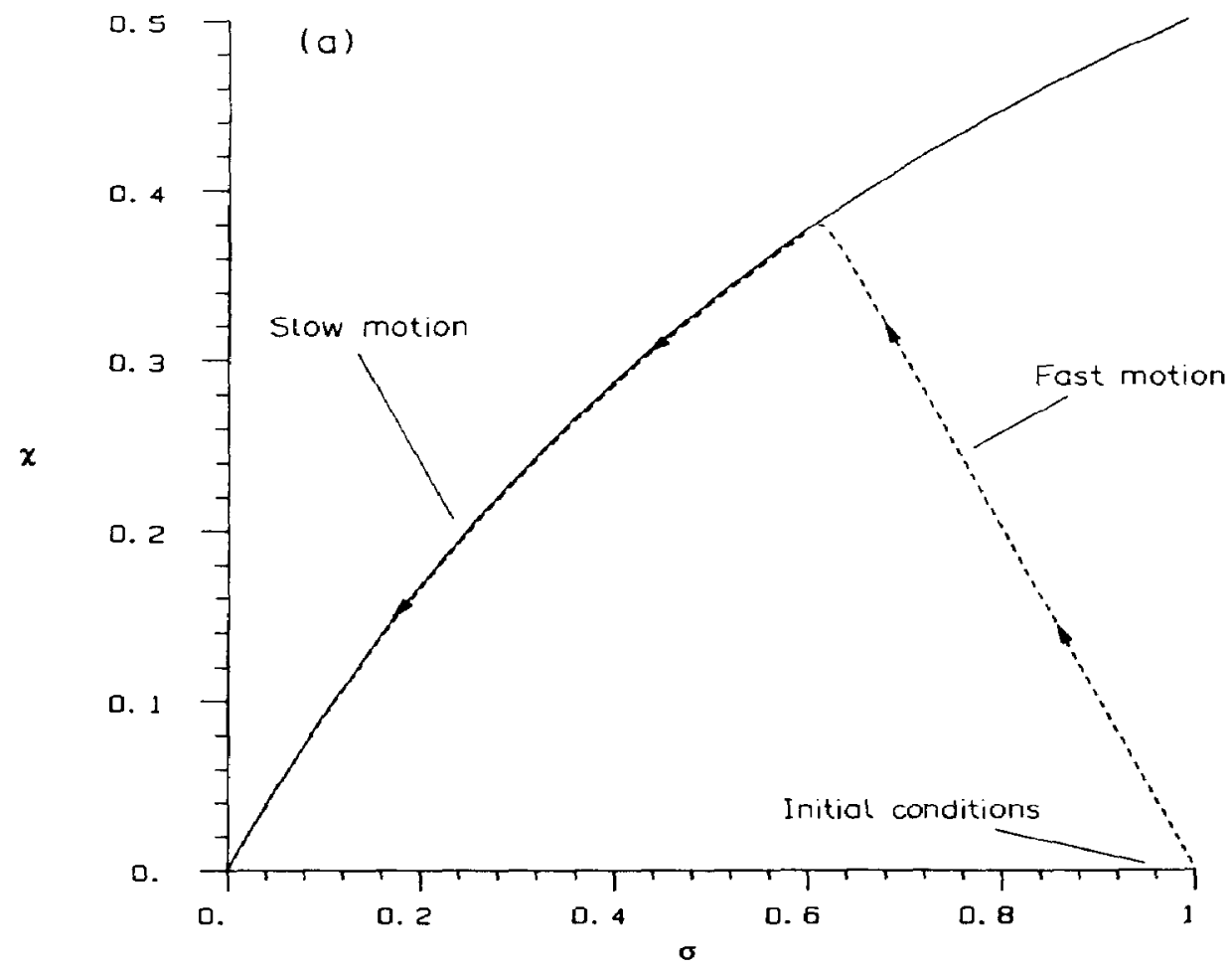

$\sigma$
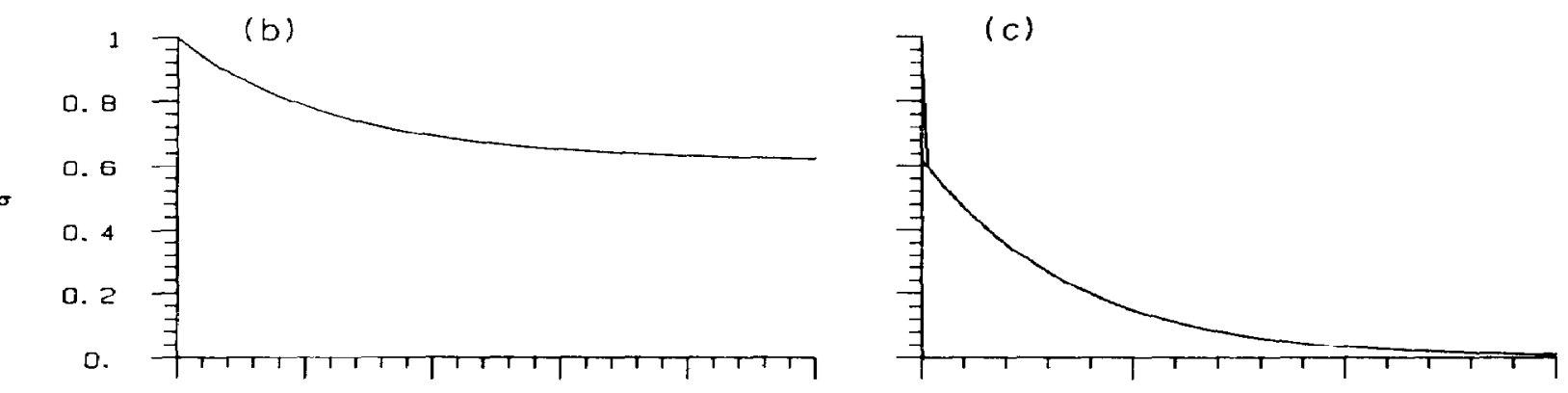

$\boldsymbol{x}$
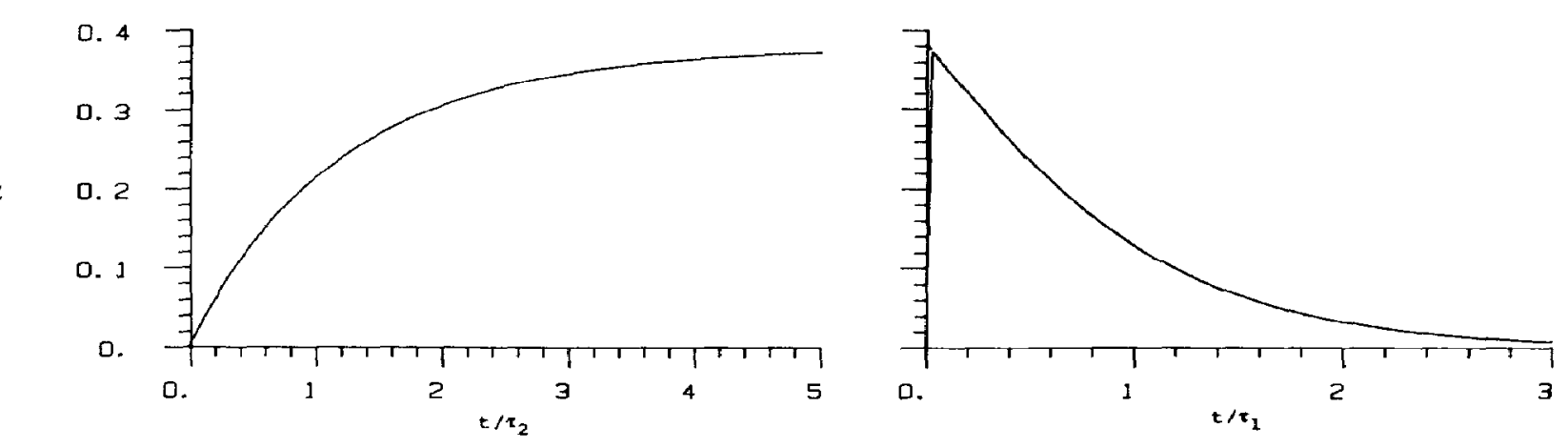

Fig. 4. Dynamic behaviour of the Michaelis-Menten reaction mechanism for $k_{2} / k_{-1}=0.01, e_{0} / K_{\mathrm{m}}=1$, $s_{0} / K_{\mathrm{m}}=1$. (a) The phase plane (solid line, quasi-equilibrium solution; dashed line, full numerical solution). (b) The fast transients. (c) The slow transients (solid line, full numerical solution; dashed line, quasiequilibrium solution). 

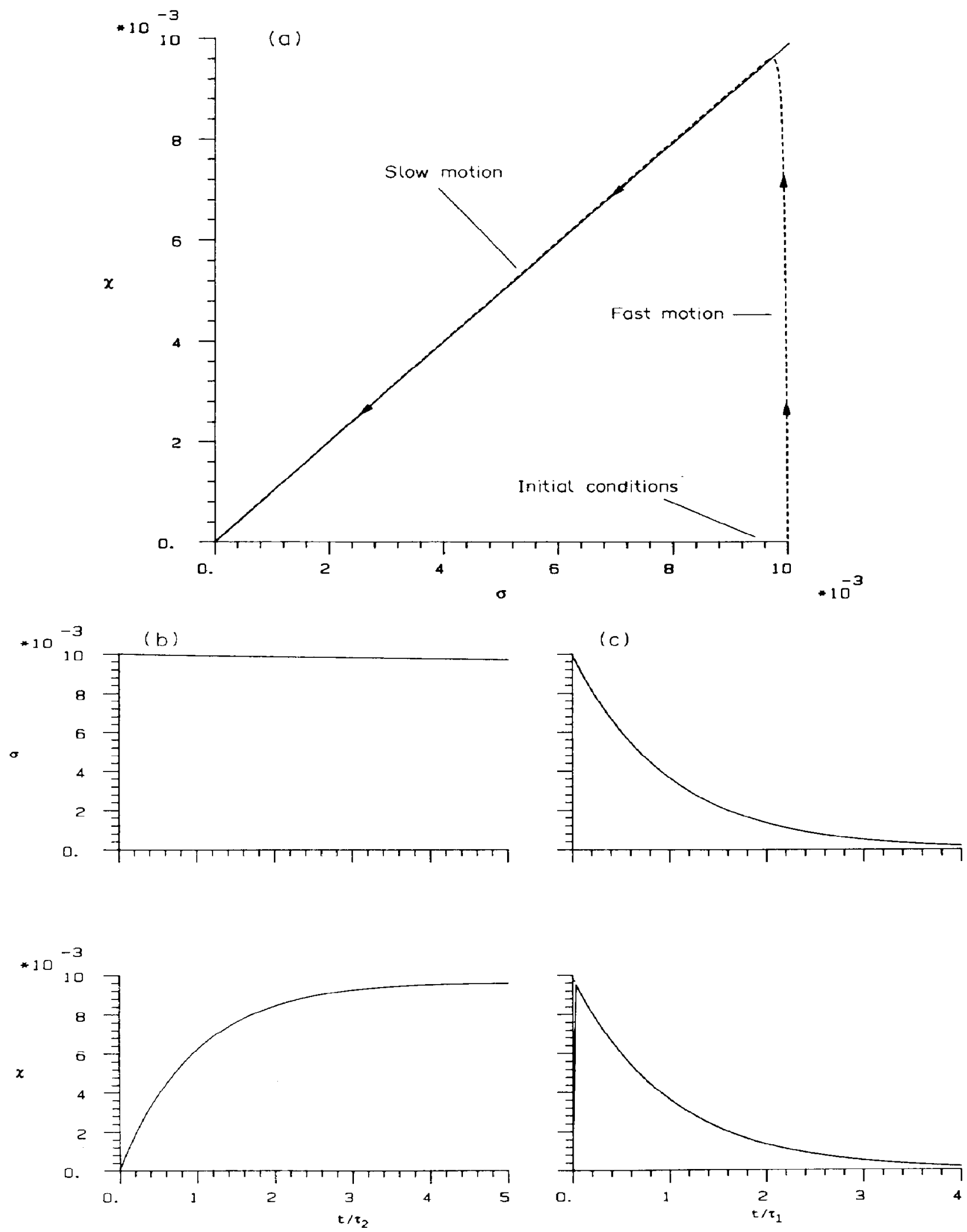

Fig. 5. Dynamic behaviour of the Michaelis-Menten reaction mechanism for $k_{2} / k_{-1}=1, e_{0} / K_{m}=0.01$, $s_{0} / K_{m}=0.01$. (a) The phase plane (solid line, quasi-steady-state solution; dashed line, full numerical solution); (b) The fast transients. (c) The slow transients (solid line, full numerical solution; dashed line, quasi-steady-state solution). 
tration of the enzyme can approach the $K_{\mathrm{m}}$ value and the quasi-steady-state solution could lead to erroneous results. Further discussion is found in Palsson (1984).

Crooke et al. (1979) have discussed the importance of the ratio $e_{0} / K_{m}$ for Michaelis-Menten kinetics. Their analysis showed that when the ratio $e_{0} / K_{m}$ is small, the quasi-steady-state assumption holds and when it is large the quasi-equilibrium assumption is valid. This agrees with our results for limits (a) and (c), respectively. In a wider context, the $e_{0} / K_{m}$ ratio can be interpreted as a measure of the relative magnitudes of $\mathrm{d} s / \mathrm{d} t$ and $\mathrm{d} x / \mathrm{d} t$; this is evident when one looks at the composition of the fast mode in limits (a) and (c).

The quasi-equilibrium assumption holds if $e_{0} \gg K_{m}$ or when $k_{2} \ll k_{-1}$ [limits (c) and (d), respectively]. The condition $k_{2} \ll k_{-1}$ is a kinetic one and will not change with the total concentrations $e_{0}$ and $s_{0}$ : it is an intrinsic kinetic property of the enzyme. The above results show that appropriate use of the quasi-equilibrium assumption to describe the motion of the substrate must include adjustment of the initial conditions. Otherwise erroneous results follow from the analysis of initial rate data.

Acknowledgement - The author is gratcful for the help that Thomas Groshans gave to complete this manuscript.

\section{NOTATION}

$a$

$b$ dimensionless group, $e_{\mathrm{o}} / K_{\mathrm{m}}$

c dimensionless group, $s_{0} / K_{m}$

$c_{1}, c_{2}$ independent elements of the eigenrows

$e$ concentration of the free enzyme

E free enzyme species

$f \quad$ general non-linear function

$j_{i k} \quad i k$-th element of the Jacobian matrix

$J$ Jacobian matrix

$k_{j} \quad$ kinetic constant

$K_{\mathrm{m}} \quad$ Michaelis constant, $\left(k_{2}+k_{-1}\right) / k_{1}$

$K_{s} \quad$ dissociation constant, $k_{-1} / k_{1}$

$m_{1}, m_{2}$ normal modes

$p \quad$ concentration of the product

P product

$s \quad$ concentration of the substrate

S substrate

$t$ time

$\mathbf{u}_{1}, \mathbf{u}_{2}$ eigenrows

$x$ concentration of the intermediate complex

$X$ substrate enzyme intermediate complex

y concentration vector

\section{Greek letters}

$\lambda_{1}, \lambda_{2}$ eigenvalues of the Jacobian matrix

$\sigma \quad$ dimensionless substrate concentration, $s / K_{\mathrm{m}}$

$\tau$ dimensionless time, $k_{1} e_{0} t$

$\chi$ dimensionless intermediate complex concentration, $x / e_{0}$

\section{Subscripts}

ref reference state qss quasi-steady state

qe quasi-equilibrium

0 initial condition

\section{Superscript \\ t transpose}

\section{REFERENCES}

Albery, W. J. and Knowles, J. R., 1976, Evolution of enzyme function and development of catalytic efficiency. Biochemistry 15, 5631-5640.

Albery, W. J. and Knowles, J. R., 1977, Efficiency and evolution of enzyme catalysis. Angew. Chem. Int. Ed. Engl. 16, 285-293.

Bowen, J. R., Acrivos, A. and Oppenheim, A. K., 1963, Singular perturbation refinement to quasi-steady-state approximation in chemical kinetics. Chem. Engng $S c i .18$, $177-188$.

Briggs, G. E. and Haldane, J. B. S., 1925, A note on the kinetics of enzyme action. Biochem. J. 19, 338-339.

Byrne, G. D. and Hindmarsh, A. C., 1975, A polyalgorithm for the numerical solution of ordinary differential equations. ACM Trans. math. Software 1, 71-96.

Chance, B., 1943, The kinctics of the enzyme-substrate compound of peroxidase. $J$. biol. Chem. 151, 553-577.

Cleland, W. W., 1975, What limits the rate of an enzymecatalyzed reaction? Acc. Chem. Res. 8, 145-151.

Crooke, P. S., Tanner, R. D. and Aris, R., 1979, The role of dimensionless parameters in the Briggs-Haldane and Michaelis-Menten approximations. Chem. Engng Sci. 34, $1354-1357$.

Darvey, I. G., Klotz, A. H. and Ritter, M., 1978a, On the kinetics of enzyme action. Bull. Math. Biol. 40, 727-734.

Darvey, I. G., Klotz, A. H. and Ritter, M., 1978b, On the kinetics of consecutive, bimolecular reactions. Aust. $J$. Chem. 31, 693-694.

Hammes, G. G. and Schimmel, P. R., 1970, Rapid reactions and transient states. In The Enzymes (Edited by Boyer, P. D.), pp. 67-114. Academic Press, New York.

Heineken, F. G., Tsuchiya, H. M. and Aris, R., 1967, On the mathematical status of the pseudo-steady-state hypothesis in biochemical kinetics. Math. Biosci. 1, 95-113.

Henri, V., 1903, Lois Generales de l'Action des Diastases. Hermann, Paris.

Hill, C. M., Waight, R. D. and Bardsley, W. G., 1977, Does any enzyme follow the Michaelis-Menten equation? Molec. cell. Biochem. 15, 173-178.

Hommes, F. A., 1962, The integrated Michaelis-Menten equation. J. Chem. Phys. 26, 271-273.

Masters, C. J., 1977, Metabolic control and the microenvironment. Curr. Top. cell. Reg. 12, 75-105.

Meiske, $W$., 1978, An approximate solution of the Michaelis-Menten Mechanism for quasi-steady state and quasi-equilibrium. Math. Biosci. 42, 63-71.

Michaelis. L. and Menten, $M$, 1913, Die Kinetik der Invertinwirkung. Biochem. Z. 49.333-369.

Palsson, B. O., 1984, Mathematical modelling of dynamics and control in metabolic networks. Ph.D. Thesis, University of Wisconsin-Madison.

Palsson, B. O. and Lightfoot, E. N., 1984, Mathematical modelling of dynamics and control in metabolic networks. Part I: On Michaelis-Menten kinetics. J. theor. Biol. 111, $273-321$

Segal, H. L., 1959, The development of enzyme kinetics. In The Enzymes (Edited by Hoyer, P. D., Lardy, H. and Myrback, K.) 2nd edn, Vol. 1, pp. 1-48. Academic Press, New York.

Srere, P. A., 1967, Enzyme concentrations in tissues. Science 158, 936-937.

Srere, P. A., 1970, Enzyme concentrations in tissues-II Biochem. Med. 4, 43-46. 